\title{
Elucidating Molecular Mechanisms of Mitochondrial Fission Machinery via Helical Studies of Drp1 and its Partner Proteins
}

\author{
Kyle T. Whiddon ${ }^{1 *}$, Ryan W. Clinton ${ }^{1}$ and Jason A. Mears ${ }^{1}$ \\ 1. Department of Pharmacology, Center for Mitochondrial Diseases, the Cleveland Center for Membrane \\ and Structural Biology, Case Western Reserve University School of Medicine, Cleveland, OH, USA. \\ * Corresponding author: kxw415@case.edu
}

Regulation of mitochondrial fission plays a significant role in cellular energy distribution, apoptosis, and autophagy of damaged mitochondrial components, all of which in turn impacts proper cellular function [1]. Dynamin related protein 1 (Drp1) has been shown to be an essential protein involved in mammalian mitochondrial fission, with homologs being found in all eukaryotes [2, 3, 4]. Mutations in Drp1 have also been shown to cause neurological disorders, owing to its importance in the process of segregating mitochondria [1].

It is known that Drp1 dimers are recruited to the mitochondrial outer membrane (MOM) from the cytosol, where they are commonly localized, and form an oligomeric complex that can initiate membrane constriction [5]. Key protein partners in this recruitment include mitochondrial fission factor (Mff), mitochondrial dynamics proteins of 49 and $51 \mathrm{kDa}(\mathrm{MiD}$ 49/51), and mitochondrial fission 1 protein (Fis1) [6]. Lipid interactions are likely important for recruitment and subsequent membrane remodeling, and cardiolipin has been shown to impact the assembly of Drp1 polymers [7, 8]. Still, the specific interactions and key roles of these protein and lipid factors are not yet fully understood. For example, it is not definitively known whether the functions of scaffold proteins Mff and MiD 49/51 are co-regulatory or if they are functionally different. A more complete understanding of these interactions will provide insight into their specific functions and identify molecular features that promote mitochondrial fission.

To facilitate membrane constriction, the Drp1 forms helical oligomers either alone, in the presence of lipid templates, or in the presence of partner proteins (Fig. 1). Our group and others have studies these distinct Drp1 self-assemblies using electron microscopy [9, 10, 11]. Collectively, these structural studies provide valuable insight into how these proteins and lipids interact, identifying unique conformations mediated by interactions at distinct sites. Furthermore, Drp1 is a large GTPase, and enzymatic activation through GTP addition has been shown to constrict lipid templates. The impact of protein and lipid interactions on GTPinduced constriction will also be examined to explore conformational changes during membrane constriction. By investigating these interactions and conformational changes we can gain an increased understanding of how mitochondrial fission is promoted and regulated.

To investigate these helical polymers, we use cryo-electron microscopy (cryo-EM) and tomography (cryoET) techniques. Iterative helical real space reconstruction (IHRSR, [13]) is initially used to identify the helical rise and twist for distinct polymers. These parameters are then used to create 3D reconstructions, and subsequent analyses explore interactions that facilitate assembly of the fission machinery. By studying different combinations of Drp1, partner proteins, and lipids, we hope to gather detailed information about the interplay of these components during mitochondrial scission events, including potential modes of regulation and important intermediates. 
References:

[1] H Chen and DC Chan, Hum Mol Genet 18 (2009), p. R169.

[2] E Smirnova et al., J Cell Biol 143 (1998), p. 351.

[3] E Smirnova et al., Mol Biol Cell 12 (2001), p. 2245.

[4] JE Lee et al., Nat 540 (2016), p. 139.

[5] F Kraus and MT Ryan, Cell Sci 130 (2017), p. 2953.

[6] R Ramachandran, Semin Cell Dev Biol 76 (2018), p. 201.

[7] CA Francy et al., Sci Rep 7 (2017), p. 10744.

[8] N Stepanyats et al., Mol Biol Cell 26 (2015), p. 3104.

[9] R Kalia et al., Nat 558 (2018), p. 401.

[10] RW Clinton et al., J Biol Chem 291 (2016), p. 478.

[11] PJ Macdonald et al., Mol Biol Cell 25 (2014), p. 1905.

[12] CA Francy et al., J Biol Chem 290 (2015), p. 11692.

[13] EH Egelman, J Struct Biol 157 (2007), p. 83.

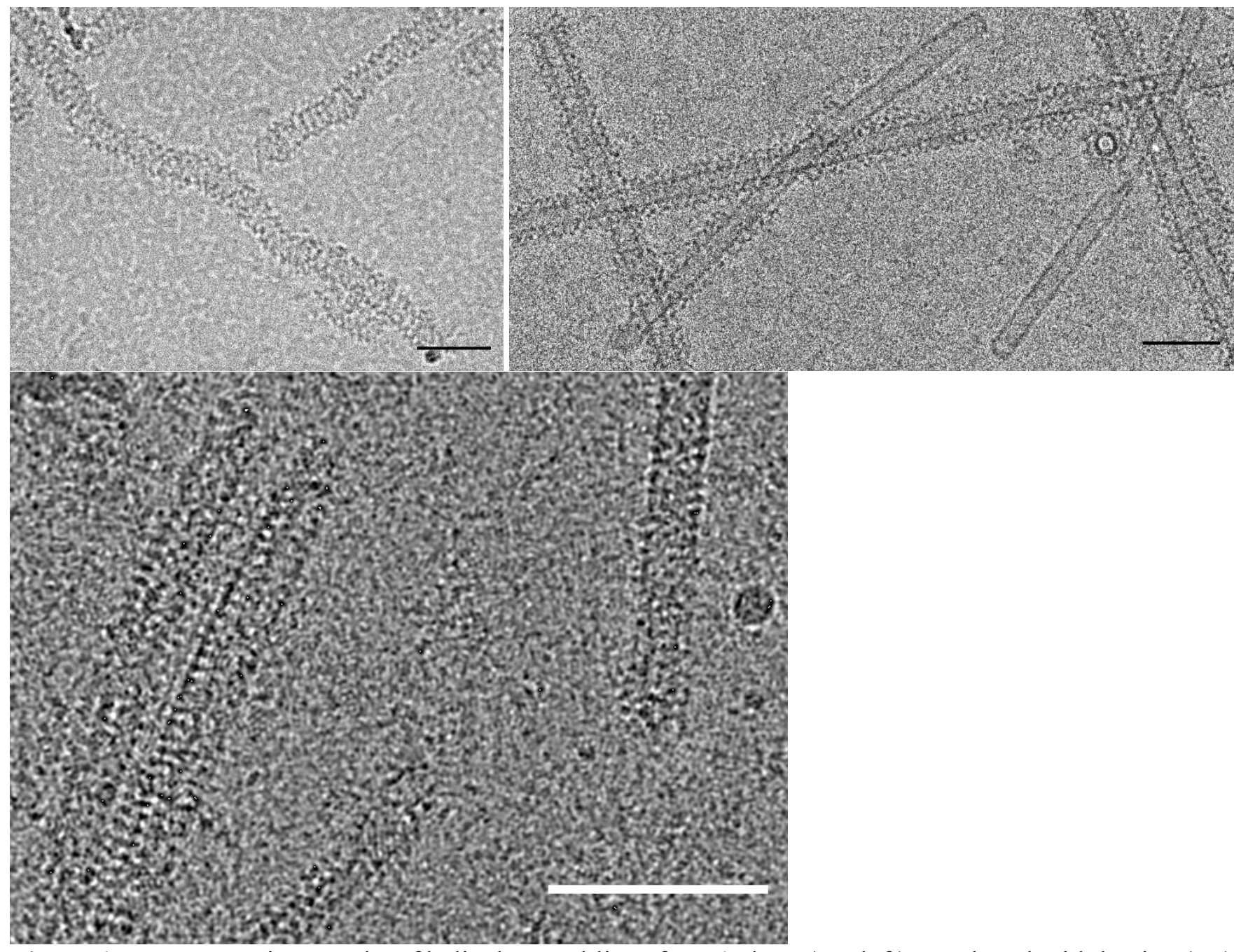

Figure 1. Cryo-EM micrographs of helical assemblies of Drp1 alone (top left), on phosphatidylserine (PS) lipid templates (top right), and in the presence of Mff (bottom). Ordered helices self-assemble, suggesting interactions that are physiologically relevant. Scale bars represent $100 \mathrm{~nm}$. 